\title{
Conditioned vestibular sway as a function of CS-UCS interval
}

JEAN A. PEZZOLI AND JOHN W. MOORE UNIVERSITY OF MASSACHUSETTS
Three groups of 18 human male Ss received vestibular sway conditioning at CS-IICS intervals of .02, .5, or $2 \mathrm{sec}$. The two shorter ISIs were most effective in combating various sources of CS-inhibition in the situation, but extinction in these groups was rapid.

Conditioning of the vestibular sway response in humans has been attempted by Revusky, Moore, \& Dzendolet (1965) and Fernald \& Moore (1966). The procedure consists of pairing a low level electrical stimulus applied to the mastoid processes with a tone. Results have indicated that, following a short series of paired trials, postural sway to the tone alone exceeds control levels. The present investigation compared conditioned vestibular sway under CS-UCS intervals (ISI) of $.02, .5$, and 2 sec. in a delayed conditioning paradigm. Method

The apparatus and procedures have been described in the two earlier reports. Briefly, postural sway was recorded by having the $S$ stand on a low platform supported by a fulcrum and two steel bars. Lateral sway exerted force on one underlying bar while withdrawing force from the contralateral bar. Strain gages affixed to these bars formed two legs of a Wheatstone bridge, the output of which provided a continuous oscillographic record. The platform was relatively stable and provided little sensation of rocking. Ss were blindfolded and remained standing on the platform for the entire experimental session. They were instructed to maintain a standard posture: heels together, knees relaxed, equal weight on both legs, and hands clasped in front. The purpose of the experiment was described as "recording brain waves to sounds under mild stress." The instructions presumably explained the presence of fluid electrodes on the S'S mastoid processes and the requirement of standing for a long period of time, while masking the experiment's true purpose.

Eighteen male undergraduate volunteers were randomly assigned to each of the three conditioning groups. These Ss received a total of 39 trials in the following sequence: three CS-alone presentations, eight blocks of four trials consisting of three CS-US trials followed by a CS-alone test trial, and four additional CS-alone trials as an extinction series. The intertrial intervals were a constant 70 sec. A control group, consisting of 18 males, also received 39 trials or CS-presentations, but the UCS was never presented. All Ss were allowed a brief period halfway between all trials to stretch and readjust their posture.

The CS was a 1000 cps tone of $70 \mathrm{db}$ SPL delivered over a loud-speaker, together with a white masking noise of $70 \mathrm{db}$ SPL which was on for the entire experimental session. The UCS was a .4 cps sinusoidal electrical stimulus of 5 sec. duration provided by a Hewlett-Packard (Model 202A) low frequency function generator. Its intensity was $.25 \mathrm{ma}$, measured from baseline $(\sin x=0)$ to peak ( $\sin x=1$ ). These parameters of the UCS are close to those found to be optimal for conditioning by Fernald \& Moore (1966). This level of stimulation is too low to seriously threaten the S's equilibrium, and despite the fact that eight additional Ss were lost due to dizziness or nausea most Ss remained unaware of their swaying or the UCS.

Since the three conditioning groups differed with respect to CS-UCS interval, the duration of the CS varied from 5.02 to $7 \mathrm{sec}$., the UCS terminating with the CS on conditioning trials. The duration of the tone in the CSalone group was a constant 5.5 sec. Since trial duration varied, response amplitude was based on different pretrial and on-trial scoring intervals. Response amplitude was measured in $\mu \nu$ as the difference between the greatest positive and negative excursions of the recording pen.

Results

The dependent variable of primary interest was the difference between on-trial and pretrial amplitudes for CS-alone trials. These data are plotted in Fig. 1

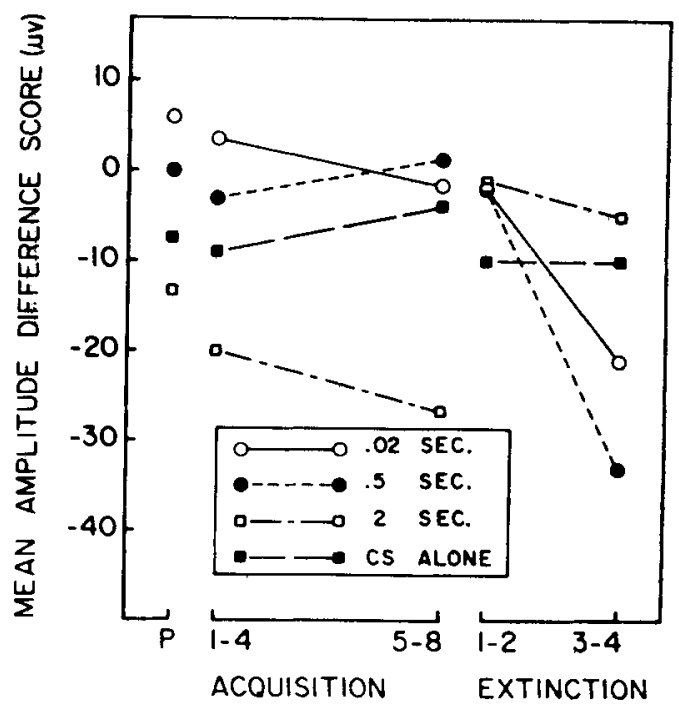

Fig. 1. Mean amplitude differences (on-trial amplitude minus pretrial amplitude) on CS alone test trials for each group. 
which shows the mean amplitude difference scores for each group averaged over (a) the three initial test trials (P), (b) acquisition test trials $1-4,(c)$ acquisition test trials $5-8$, (d) extinction trials $1-2$, and (3) extinction trials 3-4. The mean difference scores for CS-UCS trials (UCRs) were 36.2, 26.9, and $36.9 \mu \mathrm{v}$ for the $.02, .5$ and $2 \mathrm{sec}$. ISI groups, respectively. Scores on acquisition test trials tended to be negative, indicating inhibition of sway to the CS. This decrement of on-trial sway compared with CS-UCS trials was highly 'significant, $F=49.19$, df $=1 / 51, p<.001$, but the extent of this effect depended on ISI condition, $F=2.92$, $\mathrm{df}=2 / 51, \mathrm{p}<.10$. As shown in Fig. 1 , swaying on acquisition test trials was markedly lower in the $2 \mathrm{sec}$. ISI group than in the other groups. This inhibition to the CS may have been due partially to a lower starting point, as suggested by the mean difference score of -13.3 $\mu \mathrm{v}$ on initial test trials, but the fact that the inhibitory control of the CS virtually disappeared in extinction suggests that other factors were operating as well.

Figure 1 shows a decrease in response strength over extinction trials for the .02 and $.5 \mathrm{sec}$. ISI groups. Comparable decreases did not occur in the 2 sec. group or control group. The differences in extinction among conditioning groups were significant, $F=3.28, \mathrm{df}=2 / 51$, $\mathrm{p}<.05$.

\section{Discussion}

Presumably, ISIs of .02 and $.5 \mathrm{sec}$. produced conditioning of vestibular sway. These two groups responded at a higher (less negative) level than the $2 \mathrm{sec}$. group in acquisition and maintained a small superiority over the CS-alone control group. In addition, the .02 and .5 sec. groups showed clear evidence of an increase in CS-inhibition as a function of extinction trials. Admittedly, citing rapid extinction as an indicator of conditioning may be a questionable departure from the typical view that $\mathrm{CR}$-strength and resistance to extinction are directly related.

The total pattern of results in the present experiment resembles that presented in the two earlier reports. First of all, none of the studies found an orderly rise in acquisition curves. In agreement with an earlier study with dogs by Lowenbach \& Gantt (1940), conditioned postural reactions with electrical stimulation of the vestibular apparatus evidently occurs with very few trials. Secondly, it seems clear that the mere presentation of a tonal CS results in some inhibition of sway, and this inhibition is enhanced temporarily by noncontiguous presentation of the UCS (a 2 sec. ISI in the present experiment). In the Revusky et al study, for example, difference scores for the CS-alone group were close to those of the conditioning groups (ISI $=.5$ sec.), as in the present study, while inhibition to the tonal CS was marked in two control groups which received UCS presentation, but unpaired with the CS. Thus, the conditioned sway response must overcome at least two sources of inhibition, that inherent in the tonal CS and the enhancement of CS-inhibition by the addition of the UCS to the situation. The stronger CRs tend to fight this inhibition, but undergo rapid extinction with the cessation of reinforcement.

Since a ISI of .5 sec. is often optimal for conditioning, the fact that UCR magnitudes were smallest at this ISI suggests the presence of a learned inhibitory process which possibly inhibits sway according to principles recently elaborated by Kimmel (1966). Although differences in mean UCR magnitude were not significant, the fact that extinction was most dramatic in the $.5 \mathrm{sec}$. group tends to confirm the presence of this third inhibitory factor.

\section{References}

Fernald, C. D., \& Moore, J. W. Vestibular sway: parameters of the eliciting stimulus. Psychon. Sci.. 1966, 4, 55-56.

Kimmel, H. D. Inhibition of the unconditioned response in classical conditioning. Psychol. Rev., 1966, 73, 232-240.

Lowenbach, H., \& Gantt, W. H. Conditioned vestibulat reactions. J. Neurophysiol., 1940, 3, 43-48.

Revusky, B. T. L., Moore, J. W., \& Dzendolet, E. Conditioning of the human vestibular sway response. Percept. mot. Skills, 1965. 20. 593-600. 